\title{
RESEARCH
}

Open Access

\section{Cross-sectional and longitudinal associations between physical activity, sedentary behaviour and bone stiffness index across weight status in European children and adolescents}

Lan Cheng ${ }^{1,2}$, Hermann Pohlabeln', Wolfgang Ahrens ${ }^{1,2}$, Fabio Lauria ${ }^{3}$, Toomas Veidebaum ${ }^{4}$, Charalambos Chadjigeorgiou ${ }^{5}$, Dénes Molnár ${ }^{6}, G^{\prime}$ Gabriele Eiben ${ }^{7}$, Nathalie Michels ${ }^{8}$, Luis A. Moreno ${ }^{9}$, Angie S. Page ${ }^{10}$, Yannis Pitsiladis ${ }^{11}$, Antje Hebestreit ${ }^{1 *}$ and On behalf of the IDEFICS and I. Family Consortia

\begin{abstract}
Background: The associations between physical activity (PA), sedentary behaviour (SB) and bone health may be differentially affected by weight status during growth. This study aims to assess the cross-sectional and longitudinal associations between PA, SB and bone stiffness index (SI) in European children and adolescents, taking the weight status into consideration.

Methods: Calcaneus SI was first measured by quantitative ultrasound among children aged 2-9years old in 2007/ 08. It was measured again after 2 years in the IDEFICS study and after 6 years in the I. Family study. A sample of 2008 participants with time spent at sports clubs, watching TV and playing computer/games self-reported by questionnaire, and a subsample of 1037 participants with SB, light PA (LPA) and moderate-to-vigorous PA (MVPA) objectively measured using Actigraph accelerometers were included in the analyses. Weight status was defined as thin/normal and overweight/obese according to the extended International Obesity Task Force criteria. Linear mixed-effects models were used to estimate the cross-sectional and longitudinal associations between PA, SB and SI percentiles, stratified by weight status.

\footnotetext{
* Correspondence: hebestr@leibniz-bips.de

'Leibniz Institute for Prevention Research and Epidemiology - BIPS, Achterstraße 30, 28359 Bremen, Germany

Full list of author information is available at the end of the article
}

(c) The Author(s). 2020 Open Access This article is licensed under a Creative Commons Attribution 4.0 International License, which permits use, sharing, adaptation, distribution and reproduction in any medium or format, as long as you give appropriate credit to the original author(s) and the source, provide a link to the Creative Commons licence, and indicate if changes were made. The images or other third party material in this article are included in the article's Creative Commons licence, unless indicated otherwise in a credit line to the material. If material is not included in the article's Creative Commons licence and your intended use is not permitted by statutory regulation or exceeds the permitted use, you will need to obtain permission directly from the copyright holder. To view a copy of this licence, visit http://creativecommons.org/licenses/by/4.0/. The Creative Commons Public Domain Dedication waiver (http://creativecommons.org/publicdomain/zero/1.0/) applies to the data made available in this article, unless otherwise stated in a credit line to the data. 
(Continued from previous page)

Results: The cross-sectional association between weekly duration of watching TV and SI percentiles was negative in thin/normal weight group $(\beta=-0.35, p=0.008)$. However, baseline weekly duration of watching TV $(\beta=-0.63, p=$ $0.021)$ and change after 2 years $(\beta=-0.63, p=0.022)$ as well as the change in weekly duration of playing computer/games after 6 years $(\beta=-0.75, p=0.019)$ were inversely associated with corresponding changes in SI percentiles in overweight/obese group. Change in time spent at sports clubs was positively associated with change in SI percentiles after 2 years $(\beta=1.28, p=0.001)$, with comparable effect sizes across weight status. In the subsample with accelerometer data, we found a positive cross-sectional association between MVPA and SI percentiles in thin/normal weight group. Baseline MVPA predicted changes in SI percentiles after 2 and 6 years in all groups.

Conclusions: Our results suggested the beneficial effect of PA on SI. However, the increasing durations of screenbased SB might be risk factors for SI development, especially in overweight/obese children and adolescents.

Keywords: Physical activity, Sedentary behaviour, Overweight, Bone stiffness index, Observational study

\section{Background}

Bone strength is influenced by mass, architecture and density, while the trajectory of bone strength accrual persists up to the age of about 18 years until peak bone mass $(\mathrm{PBM})$ is reached $[1,2]$. Even though $\mathrm{PBM}$ is mainly explained by genetic determinants [3], it is also influenced by lifestyle-related factors such as mechanical loading, physical activity (PA), sedentary behaviour (SB) and nutrition $[4,5]$. Further, PBM is an important predictor of osteoporosis in adults, due to the age-related bone loss that occurs over time [6]. Hence, in order to prevent fractures and osteoporosis in later life, it is important to initiate preventive measures during childhood and adolescence.

The positive osteogenic effect of PA, in particular weight-bearing exercises (WBEs), on bone strength seems to be irrefutable $[7,8]$. However, despite these proven health benefits, the secular trend of PA shows a decrease among European children and adolescents, with most of them not meeting the World Health Organization (WHO) recommendations for PA [9-11]. Together with the decrease of PA, high levels of $\mathrm{SB}$ among this population group now constitute a serious public health concern [12]. In recent studies, the total duration of SB among 10- to 12-year-old European children was reported to be nearly $8 \mathrm{~h}$ per day [13], and they were also observed to spend more than $2 \mathrm{~h}$ per day in front of computer or TV screens [14]. The debate on the detrimental effects of SB on bone strength is, however, more controversial compared to beneficial effects of PA. Previous studies reported a negative [15] or null [16] association between the total duration of objectively measured SB using accelerometers and bone strength, while others suggested that self-reported screen-based SB such as using the internet [17], watching TV [18] and total screen time [19] may inversely influence bone mass. Currently, more studies are needed to combine self-reported data with objectively measured data when examining the short- and long-term effects of context-specific PA and SB on bone strength in young populations.

On one hand, sedentary lifestyles may be associated with poor bone health and are also linked to a higher risk of overweight and obesity in children and adolescents [20]. On the other hand, previous studies indicated that overweight or obese children have higher bone mass [21] or strength [22] compared to their normal weight peers, which, however, is in conflict with the unfavourable effects of sedentary lifestyles. In addition, being overweight has been reported to increase the risk for sedentary lifestyles [23], thereby leading to poor bone health. In view of these potential pathways, the associations between PA, SB and bone strength may be differentially influenced by overweight and obesity.

Understanding which specific dimensions of PA and SB influence the growing skeleton is crucial for the development of effective and sustainable strategies for increased bone strength. Particularly the role of weight status in these associations is still poorly understood. In an effort to fill this gap, information on the bone stiffness index (SI) measured using quantitative ultrasound (QUS) as a proxy indicator for bone strength has been repeatedly collected in a sample from the IDEFICS (Identification and prevention of dietary- and lifestyleinduced health effects in children and infants) and I. Family studies. Self-reported time spent at sports club, WBEs, watching TV and playing computer/games, as well as objectively measured SB, light PA (LPA) and moderate-to-vigorous PA (MVPA) were also collected to assess the cross-sectional and longitudinal associations of various kinds of PA and SB on SI in European children and adolescents across different weight statuses.

\section{Methods}

\section{Study sample}

The IDEFICS/I.Family study is the largest prospective child cohort in Europe with repeated measurements of 
anthropometric indicators, clinical examinations as well as extensive questionnaire-based information on sociodemographic factors, PA and nutrition [24, 25]. The first two waves of data collection occurred in the context of the IDEFICS study, which comprised 16,229 children from eight European countries (Belgium, Cyprus, Estonia, Germany, Hungary, Italy, Spain and Sweden), who were aged 2-9.9 years at baseline between September 2007 and May 2008. The second wave between September 2009 and May 2010 included a follow-up of 11, 043 children from baseline and 2543 newly recruited children. The third wave was conducted in the context of the I. Family study between January 2013 and June 2014 with a follow-up of 7117 children from the original IDEFICS cohort and 2501 newly recruited children. All parents provided signed informed consent for their children prior to all exanimations. In addition, children younger than 12 years gave their oral consent and children above 12 years provided a signed simplified form of consent. Ethical approval for the study was obtained from the ethics committees for participating centres in each country.

\section{Inclusion and exclusion criteria}

QUS measurements were obtained as an optional module in a subgroup of participants in the IDEFICS study, while in the I. Family study, QUS data were only available for five of the eight participating countries. We assumed that no substantial selection effects occurred since the reduced participation was mainly because of budgetary constraints and device feasibility. In order to simultaneously investigate cross-sectional and longitudinal associations between the exposures of interest and bone SI, we included 3422 children with baseline and at least one follow-up QUS measurements of both the left and right foot. In accordance with findings from a previous IDEFICS study on QUS measurement precision, there was a significant discrepancy in SI difference between the left and right foot across devices in each participating centre (unpublished data). In order to control for this discrepancy, 200 children whose QUS measurements had an SI difference between the left and right foot above 41 units (97th percentile, calculated based on 7612 repeated measurements in total) were excluded. A further 43 children who at baseline reported having medical conditions preventing participation in regular $\mathrm{PA}$ and/or known to influence bone metabolism were excluded [26].

Finally, children without self-reported PA, screenbased SB or covariate data were excluded, leaving a total of 2008 participants for the full analysis. The mean age of the main sample was 6.14 years $(\mathrm{SD}=1.80), 54.1 \%$ were boys and the proportions of low, medium and high familial socio-economic status (SES) were 10.4, 56.3 and
$33.3 \%$, respectively. A subsample of 1037 participants who provided objectively measured accelerometer-based SB, LPA and MVPA data was also analysed. The mean age of the subsample was 6.45 years $(\mathrm{SD}=1.72), 50.4 \%$ were boys and the proportions of low, medium and high familial socio-economic status (SES) were 9.4, 60.3 and $30.3 \%$, respectively. Compared with the original IDEFICS study sample, the children in both analytic samples were older (vs. 6.01 years, $\mathrm{SD}=1.79$ ) and more children had low (vs. 9.0\%) and medium levels (vs. 50.3\%) of SES. In addition, more boys were included in the main sample (vs. 50.8\%). The inclusion and exclusion process of participants for the final analysis is summarised in Fig. 1. No children from Cyprus were included in the analysis as they did not fulfil any of the inclusion criteria.

\section{Bone stiffness index}

QUS measurements on the left and right calcaneus were performed using Achilles Lunar Insight ${ }^{\text {tw }}$ (GE Healthcare, Milwaukee, WI, USA). The parameters of speed of sound $(\mathrm{SOS}, \mathrm{m} / \mathrm{s})$ and broadband ultrasound attenuation (BUA, dB/MHz) assessed by QUS devices reflect the velocity and attenuation of the ultrasound waves through the bone tissue, respectively. The SI value was estimated automatically by Lunar Achilles OsteoReport Software and reported as 'unit' according to the equation: $\mathrm{SI}=$ $(0.67 * \mathrm{BUA})+\left(0.28^{*} \mathrm{SOS}\right)-420$, with high SI values indicating better bone strength [27]. SOS, BUA and SI assessed by calcaneus QUS have been shown to be correlated with bone mineral content (BMC) and bone mineral density (BMD) assessed by dual energy X-ray absorptiometry (DXA) in children and adolescents in previous studies $[28,29]$. There is also evidence suggesting that QUS devices could be used to estimate fracture risk and osteoporosis in childhood [27] and adulthood [30]. Compared to DXA, the main advantages of QUS devices are that they are non-radiating, quick and costeffective, making them more suitable for large-scale epidemiological studies, particularly in healthy young populations. The SI value not only reflects bone density, it is also influenced by the architecture and elasticity of the bone tissue, which makes it possible to provide some structural information [27]. The reproducibility in each QUS device was tested on 91 children from the IDEFICS baseline; no differences were found in SI values between the three repeated measurements and between measurements at the left and right foot. The root-mean-square coefficients of variation $\left(\mathrm{CV}_{\mathrm{RMS}}\right)$ for the SI measurements were 7.2 and $9.2 \%$ on the left and right foot, respectively (unpublished data). In line with the study protocol, daily machine calibration was carried out during the entire study period and the measurements were taken by trained nurses according to the standard procedure [31]. Two different sizes of foot adapters were 


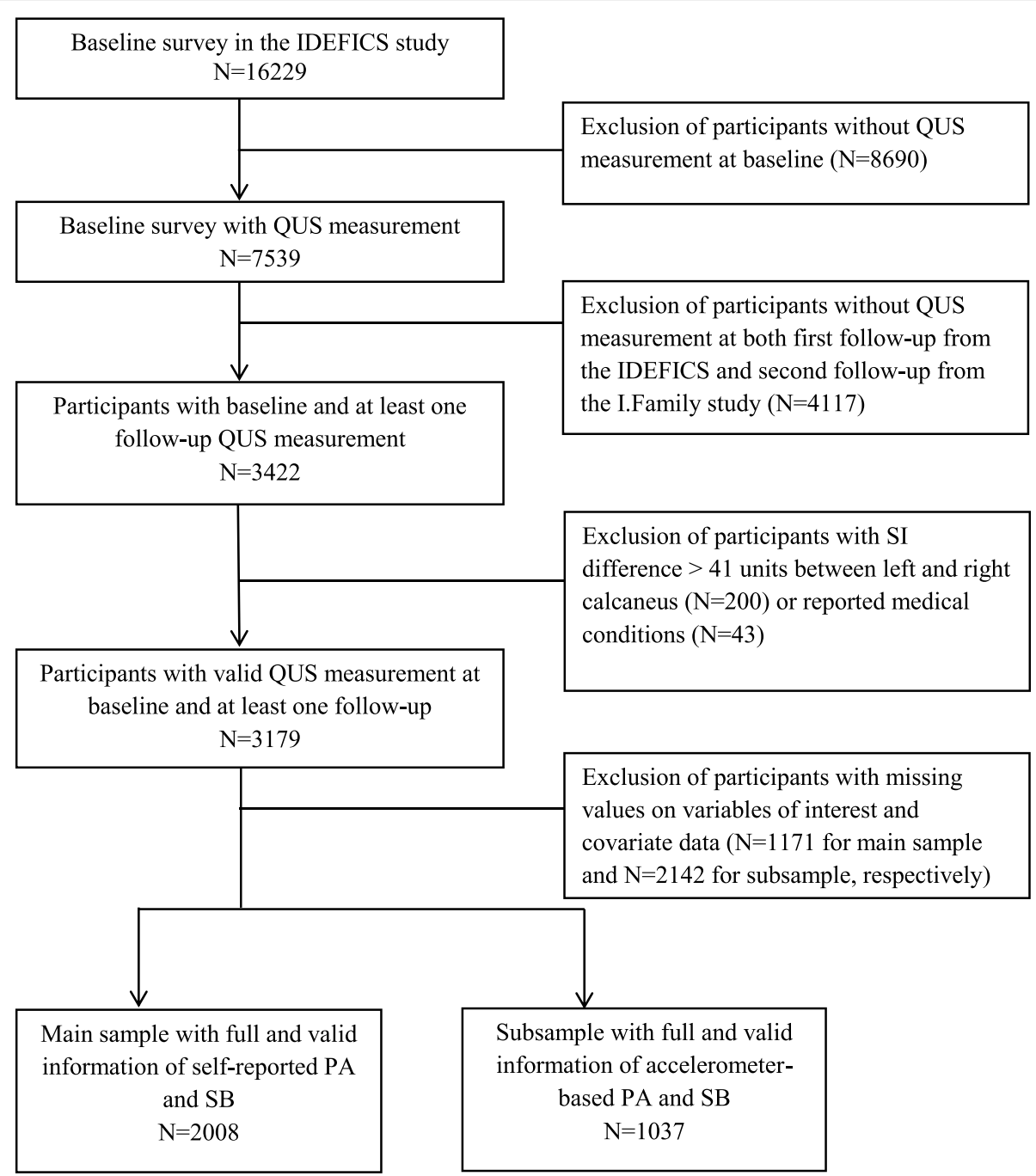

Fig. 1 Flow diagram of children included in final analysis groups, baseline examination (IDEFICS): 2007/2008, two-year follow-up examination (IDEFICS): 2009/2010, six-year follow-up examination (I.Family): 2013/2014. QUS quantitative ultrasound, PA physical activity, SB sedentary behaviour

used to put the calcaneus in an appropriate position. The mean SI of the left and right calcaneus was calculated and used in the statistical analysis. The distribution of SI was assessed and age-, sex- and height-specific percentiles for SI values were calculated as outcomes [32].

\section{Anthropometric measurements}

Height and weight were measured in light clothing without shoes. The former was measured to the nearest 0.1 cm using a standard clinical Seca 225 stadiometer (Seca, Hamburg, Germany) and the latter to the nearest $0.1 \mathrm{~kg}$ using a BC420 SMA scale (Tanita, Amsterdam, the Netherlands). The intra- and inter-observer reliability for height and weight were conducted in each centre, with CV\% ranging from 0.2 to $1.0 \%$ in the IDEFICS [33] and I. Family study (unpublished data). In all study centres, trained nurses took the measurements following standardised procedures. For each child, age- and sexspecific z-scores of height and weight were determined using the LMS method by Cole et al. [34]. Body mass index (BMI, $\mathrm{kg} / \mathrm{m}^{2}$ ) was calculated as body weight divided by squared body height, and weight status (thin/ normal and overweight/obese) was classified at the 90th percentile (passing through the BMI of 25 at the age of 18) as recommended based on the extended International Obesity Task Force (IOTF) BMI criteria [35].

\section{Questionnaires}

The questionnaires relating to lifestyle behaviours were answered by parents for young children up to 11 years old; they were self-reported for 12- to 15-year-old adolescents. The following information was collected for each child/adolescent: whether they were a member of a sports club and if so, 1) how many hours and minutes 
per week they spent there and 2) in what kind of sports they participated at the sports club. The time spent at sports clubs was calculated by adding the hours and minutes reported and expressed as hours per week (h/w). The variable WBE was based on all reported types of sports, classified according to the loads and categorised into moderate or high mechanical loads (ball games, gymnastics, dancing, skating, martial arts and athletics, etc.) and no or low mechanical loads (swimming, biking and horseback riding, etc.). In addition, information regarding the time usually spent watching TV/videos/ DVDs and playing on a computer/game console on a normal weekday and weekend day was also collected. For both questions, six response categories were offered and converted into the following scoring system: not at all $=0, \quad<30 \mathrm{~min}=1,<1 \mathrm{~h}=2,1-<2 \mathrm{~h}=3,2-3 \mathrm{~h}=4$, and $>3 \mathrm{~h}=5$. Each screen-based SB was calculated separately for weekdays and weekend days by adding the converted responses of the individual questions and expressed as hours per week (h/w). Weekly duration of watching TV was further classified into $>14 \mathrm{~h} / \mathrm{w}$ and $\leq 14 \mathrm{~h} / \mathrm{w}$ in accordance with international guidelines $[36,37]$ to investigate the benefit of fulfilling the guidelines on SI.

\section{Accelerometer data}

PA and SB were objectively measured using Actigraph accelerometer devices (Actigraph, LLC, Pensacola, FL, USA) in a subsample of participants. Parents or legal guardians were asked to ensure that their child wore the accelerometer on the right hip and that it was only removed during water-based activities and bedtime. Data were collected in the vertical axis for three-axial accelerometer. In the IDEFICS study, either the GT1M or ActiTrainer was used; the sensor units of both models are identical. In previous validation studies, both types of accelerometers have been observed to measure comparable MPA, LPA and MVPA levels [38]. However, the outputs of counts per minute (cpm) [39] and low PA levels (e.g. LPA and walking) [38] for ActiTrainer were lower than other Actigraph models, thus care should be taken when interpreting these results. In the I. Family study, either the GT1M or GT3x + was used, the comparability of the vertical axis outputs for the GT3X and GT1M has also been proven in previous studies [40, 41]. The participants were requested to wear the accelerometer for at least 3 days including one weekend day in the IDEFICS study and for 7 days in the I. Family study. Participants were included in the analyses only if they had at least 6 $h$ of data per day and three accelerometer measurement days. Further, any periods containing $20 \mathrm{~min}$ or more of consecutive zero counts were removed as non-wearing time. All accelerometer recordings were integrated over 60 s epochs and the intensity levels were classified as SB $(\leq 100 \mathrm{cpm}), \quad$ LPA $\quad(>100-<2296 \mathrm{cpm})$ and MVPA $(\geq 2296 \mathrm{cpm})$ according to the cut-off points suggested by Evenson et al. [42]. More details on processing of accelerometer data in the IDEFICS/I.Family study have been published elsewhere $[9,43]$. In the present study, total durations of objectively measured SB and LPA were expressed as hours per day $(\mathrm{h} / \mathrm{d})$. In order to better interpret the regression coefficients, the unit of objectively measured MVPA was converted to 10 min per day (10 $\mathrm{min} / \mathrm{d}$ ) according to previous studies, reporting that every additional $10 \mathrm{~min} / \mathrm{d}$ of MVPA was associated with increases of bone health indicators in children [26, 44]. We further considered the variable objectively measured MVPA as a dichotomised instead of a continuous variable. According to WHO recommended levels of PA for children and adolescents aged 5-17 years old, daily duration of objectively measured MVPA $\geq 1 \mathrm{~h} / \mathrm{d}$ was regarded as adhering to the guideline [45].

\section{Confounding variables}

Sex, age and questions regarding the familial SES of participants were reported by parents. SES was assessed based on the highest educational level of parents according to the International Standard Classification of Education (ISCED) and categorised into low (ISCED 0,1,2), medium (ISCED 3,4) and high (ISCED 5,6) [46]. The voice change of boys and the first menstrual period of girls from the age of 8 years old were collected in the I. Family study as a proxy for pubertal development and further categorised into pre-pubertal and pubertal in the present study. Both indicators have been widely used to assess maturation in previous epidemiological studies, suggesting that changes in the male voice often occur between Tanner stages 3 and 4 [47, 48], which is the comparable onset age of menarche in females [49]. The variance between countries was also considered. Further, sunlight exposure as the most important source for vitamin D synthesis was also taken into consideration [50], calculated by mean daylight duration for each examination month in each location based on astronomical tables [26].

\section{Statistical analyses}

All analyses were performed using SAS software (V9.3; SAS Institute Inc., Cary, North Carolina, USA). The changes in continuous variables were determined by calculating the differences between follow-up after 2 or 6 years and baseline values. Descriptive statistics, e.g. means, standard deviations (SD), and frequencies for baseline and changes of each variable were conducted and stratified by weight status (thin/normal and overweight/obese) in each survey. Differences for continuous variables were compared using $\mathrm{t}$ tests, and chi-square tests were used for categorical variables. 
Linear mixed-effects models were used to estimate the cross-sectional and longitudinal associations between PA, SB and SI percentiles, with country as a random effect (at the level of the intercept). To avoid getting associations that are irrelevantly statistically significant, for instance simply due to the large sample size or to multiple testing, a more stringent criterion for statistical significance $(\alpha=0.01)$ was chosen. Regression coefficients $(\beta)$ and 99\%-confidence intervals $(99 \% \mathrm{CI})$ were estimated in all models. The cross-sectional analyses were based on the data from baseline and the outcome was baseline SI percentiles. Weekly duration of watching TV, playing computer/games and sports club activities as well as WBE were taken into consideration as exposures and adjusted for sex, age, SES, daylight duration, weight and height $\mathrm{z}$-scores. In the longitudinal analyses, the outcomes were the changes in SI percentiles after 2 or 6 years, with taking the baseline and changes in exposures into consideration. In addition to the confounding factors described above, we also included baseline SI percentiles and pubertal status in the longitudinal models. Based on the same analytical approach, objectively measured SB, LPA and MVPA were considered in subgroup analyses and presented separately. All analyses were performed in the whole group and then further stratified by thin/normal and overweight/obese groups. Differences in the association between each exposure of interest and corresponding outcome across weight status were further tested by interactive terms in the whole group models, however, they were not considered in the final analyses since no statistically significant interactions were observed.

\section{Results}

\section{Descriptive characteristics of study population}

As summarised in Table 1, the baseline proportions of overweight/obese children in the main sample $(n=2008)$ and subsample with accelerometer data $(n=1037)$ were 19.0 and $19.7 \%$, respectively. At individual country-level, Italy had the highest proportion of overweight/obese children and Belgium the lowest. Regarding SI, in the main sample, the means of SI percentiles were $43.92 \pm$ 27.84 in the thin/normal weight group and $41.36 \pm 25.76$ in the overweight/obese group at baseline with an increase for both groups during the two-year and six-year follow-up periods (Table 2). Increasing trends in the weekly duration of watching TV, playing computer/ games and time spent at sports clubs were also observed after 2 and 6 years. In the subsample with accelerometer

Table 1 Demographic characteristics of the study population, stratified by weight status

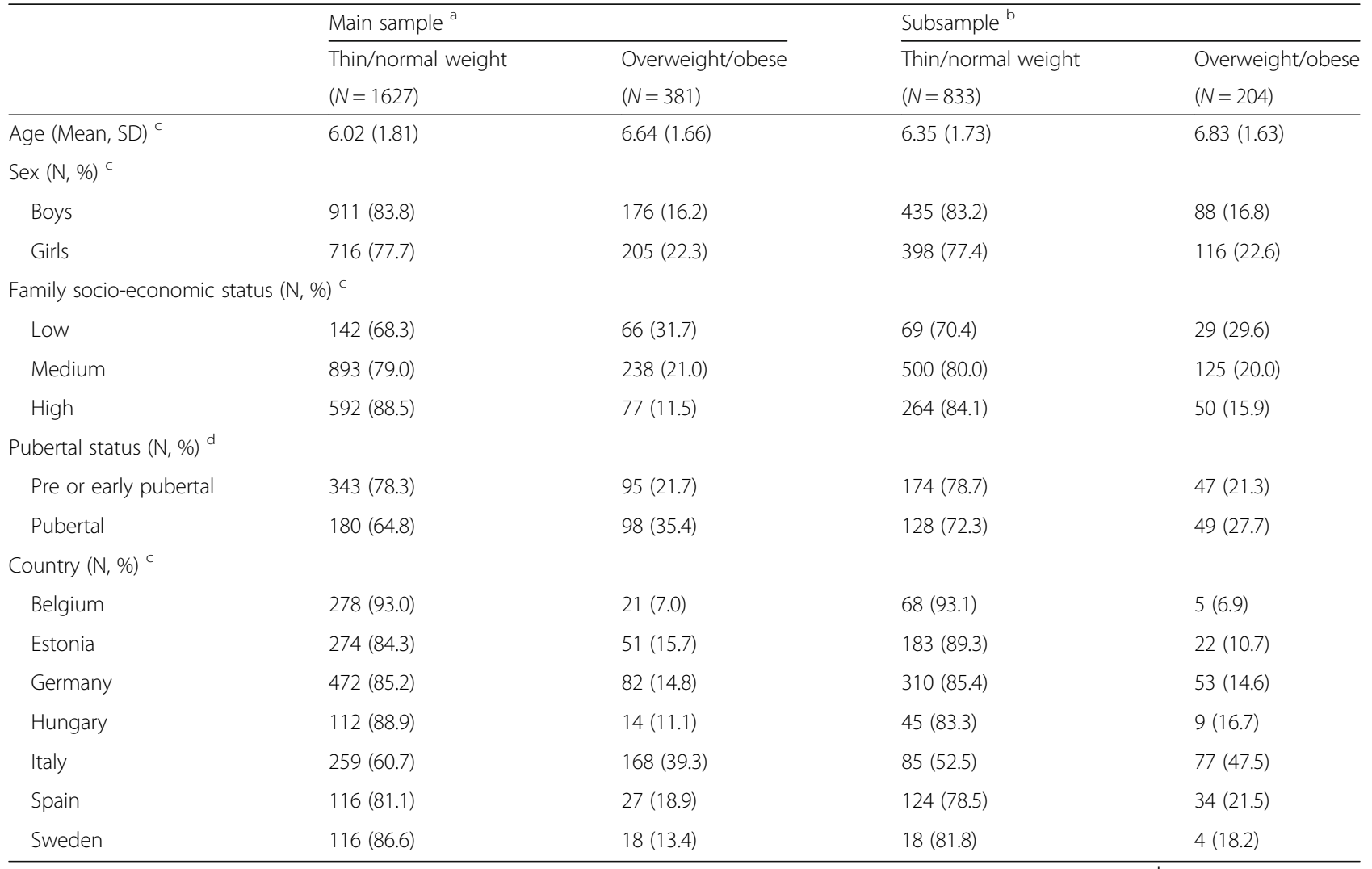

${ }^{a}$ Main sample included participants with full information of self-reported physical activity and sedentary behaviour as well as co-variables. ${ }^{b}$ Subsample included the participants with full information of accelerometer data as well as co-variables. ${ }^{c}$ Data from baseline survey; ${ }^{\mathrm{d}}$ Data from six-year follow-up survey 
Table 2 Baseline and changes of measurements over two-year and six-year follow-up

\begin{tabular}{|c|c|c|c|c|c|c|}
\hline & \multicolumn{2}{|l|}{ Baseline } & \multicolumn{2}{|l|}{ Two-year changes } & \multicolumn{2}{|l|}{ Six-year changes } \\
\hline & $\begin{array}{l}\text { Thin/normal } \\
\text { weight }\end{array}$ & $\begin{array}{l}\text { Overweight/ } \\
\text { obese }\end{array}$ & $\begin{array}{l}\text { Thin/normal } \\
\text { weight }\end{array}$ & $\begin{array}{l}\text { Overweight/ } \\
\text { obese }\end{array}$ & $\begin{array}{l}\text { Thin/normal } \\
\text { weight }\end{array}$ & $\begin{array}{l}\text { Overweight/ } \\
\text { obese }\end{array}$ \\
\hline & $\left(N=1627^{a} / 833^{b}\right)$ & $\left(N=381^{a} / 204^{b}\right)$ & $\left(N=1273^{a} / 633^{b}\right)$ & $\left(N=380^{a} / 206^{b}\right)$ & $\left(N=523^{a} / 302^{b}\right)$ & $\left(N=193^{a} / 96^{b}\right)$ \\
\hline \multicolumn{7}{|l|}{ Anthropometric measures (Mean, SD) ${ }^{c}$} \\
\hline Percentiles of bone stiffness index & $43.92(27.84)$ & $41.36(25.76)$ & $3.86(30.17)$ & $4.60(25.55)$ & $8.08(34.59)^{*}$ & $18.88(33.46)^{*}$ \\
\hline Height z-score & $0.37(1.00)^{*}$ & $0.87(1.02)^{*}$ & $0.04(0.40)$ & $0.06(0.39)$ & $0.20(0.60)^{*}$ & $0.05(0.68)^{*}$ \\
\hline Weight z-score & $0.07(0.87)^{*}$ & $2.00(0.78)^{*}$ & $0.03(0.38)^{*}$ & $0.19(0.52)^{*}$ & $0.21(0.66)^{*}$ & $0.43(0.87)^{*}$ \\
\hline \multicolumn{7}{|l|}{ Reported healthy behaviour } \\
\hline $\begin{array}{l}\text { Watching TV/video/DVD (hours/ } \\
\text { week, Mean, SD) }{ }^{c}\end{array}$ & $8.67(5.00)^{*}$ & $9.74(5.32)^{*}$ & $0.62(4.57)$ & $0.71(5.13)$ & $2.05(6.41)$ & $3.09(7.57)$ \\
\hline $\begin{array}{l}\text { Playing computer/games (hours/ } \\
\text { week, Mean, SD) }{ }^{c}\end{array}$ & $2.34(3.39)$ & $2.69(3.58)$ & $1.38(3.58)^{*}$ & $2.17(4.66)^{*}$ & $2.84(5.83)$ & $3.63(7.16)$ \\
\hline $\begin{array}{l}\text { Sports clubs (hours/week, Mean, } \\
\text { SD) }{ }^{c}\end{array}$ & $1.13(1.60)$ & $1.32(1.63)$ & $0.72(1.77)$ & $0.56(1.89)$ & $1.41(2.56)$ & $0.90(2.67)$ \\
\hline \multicolumn{7}{|l|}{ Weight bearing sports $(\mathrm{N}, \%)^{d}$} \\
\hline $\begin{array}{l}\text { Moderate or high mechanical } \\
\text { loads }\end{array}$ & $752(79.5)$ & $194(20.5)$ & $866(76.5)$ & $266(23.5)$ & $396(73.5)$ & $143(26.5)$ \\
\hline No or low mechanical loads & $875(82.4)$ & $187(17.6)$ & $407(78.1)$ & $114(21.9)$ & 127 (71.8) & $50(28.2)$ \\
\hline \multicolumn{7}{|l|}{ Accelerometer data (Mean, SD) } \\
\hline Sedentary time (hours/day) ${ }^{c}$ & $4.46(1.21)^{*}$ & $4.72(1.26)^{*}$ & $0.70(1.39)$ & $0.61(1.42)$ & $2.31(1.53)$ & $2.41(1.59)$ \\
\hline Light physical activity (hours/day) $^{c}$ & $6.37(1.00)$ & $6.39(1.10)$ & $-0.46(1.14)$ & $-0.57(1.23)$ & $-1.47(1.28)$ & $-1.56(1.29)$ \\
\hline $\begin{array}{l}\text { Moderate-to-vigorous physical } \\
\text { activity }\left(10 \mathrm{~min} / \text { day) }{ }^{c}\right.\end{array}$ & $4.28(2.21)^{*}$ & $3.57(1.93)^{*}$ & $-0.23(2.43)$ & $-0.45(1.96)$ & $0.10(2.61)$ & $-0.50(2.72)$ \\
\hline
\end{tabular}

${ }^{a}$ Sample size with full information of self-reported physical activity and sedentary behaviour as well as co-variables. ${ }^{\mathrm{b}}$ Sample size with full information of accelerometer data as well as co-variables. ${ }^{c}$ Changes of values were the differences between follow-up and baseline measurements. ${ }^{d}$ Changes of values were the percentages of reported moderate or high mechanical loads at baseline or follow-up, and no or low mechanical loads in both waves, respectively. ${ }^{*} p<0.01$

data, there was a trend of increasing SB while LPA and MVPA slightly decreased over time.

\section{Cross-sectional associations between SB, PA and SI percentiles}

No statistically significant associations between selfreported and objectively measured SB, PA and SI were observed in the whole group at baseline. However, in thin/ normal weight group, weekly duration of watching TV was inversely associated with SI percentiles $(\beta=-0.35$, $p=0.008)$, while daily duration of objectively measured MVPA was positively associated with SI percentiles $(\beta=$ $1.18, p=0.008)$. Opposite but not statistically significant associations were observed for the overweight/obese group where SI percentiles were positively associated with the weekly duration of watching TV $(\beta=0.03, p=0.906)$ while inversely associated with the daily duration of objectively measured MVPA $(\beta=-0.23, p=0.807)$ (Table 3$)$.

\section{Longitudinal effects of SB and PA on changes in SI percentiles}

In the whole group, change in time spent at sports clubs was positively associated with change in SI percentiles after
2 years $(\beta=1.28, p=0.001)$; objectively measured MVPA at baseline was a strong predictor for change in SI percentiles $(\beta=2.77, p<0.001)$. Similar effect sizes were observed after stratifying by weight status although the findings for overweight/obese group were not statistically significant. In contrast, weekly duration of watching TV at baseline $(\beta=-0.63, p=0.021)$ and change after 2 years $(\beta=-0.63, p=0.022)$ were inversely associated with change in SI percentiles only in overweight/obese group (Table 4).

Regarding the six-year follow-up, a statistically significant positive association between change in time spent at sports clubs and corresponding change in SI percentiles was observed only for the thin/normal weight group. As observed after 2 years, objectively measured MVPA at baseline also predicted change in SI percentiles after 6 years $(\beta=3.67, p<0.001)$. In contrast to the slight effect of watching TV over the two-year period in overweight/obese group, we observed that six-year change in duration of playing computer/games was negatively associated with sixyear change in SI percentiles $(\beta=-0.75, p=0.019)$ (Table 5). 
Table 3 Cross-sectional associations between sedentary behaviour, physical activity and bone stiffness index percentiles at baseline

\begin{tabular}{|c|c|c|c|c|c|c|}
\hline & \multicolumn{2}{|l|}{ Whole group } & \multicolumn{2}{|c|}{ Thin/normal weight group } & \multicolumn{2}{|c|}{ Overweight/obese group } \\
\hline & $\beta(99 \% \mathrm{Cl})$ & $p$-value & $\beta(99 \% \mathrm{Cl})$ & $\overline{p \text {-value }}$ & $\beta(99 \% \mathrm{Cl})$ & p-value \\
\hline \multicolumn{7}{|l|}{ Main sample (self-reported data, $N=2008$ ) } \\
\hline Watching TV/video/DVD (hours/week) & $-0.23(-0.53,0.06)$ & 0.044 & $-0.35(-0.69,-0.01)$ & 0.008 & $0.03(-0.60,0.66)$ & 0.906 \\
\hline Playing computer/games (hours/week) & $-0.004(-0.49,0.48)$ & 0.984 & $0.03(-0.52,0.58)$ & 0.883 & $0.03(-0.96,1.01)$ & 0.943 \\
\hline Sports clubs (hours/week) & $1.00(-0.40,2.39)$ & 0.066 & $0.50(-1.03,2.04)$ & 0.398 & $2.68(-0.58,5.95)$ & 0.034 \\
\hline \multicolumn{7}{|l|}{ Weight bearing sports } \\
\hline $\begin{array}{l}\text { Moderate or high mechanical loads vs. No or low } \\
\text { mechanical loads (reference) }\end{array}$ & $-1.80(-6.37,2.77)$ & 0.310 & $-0.13(-5.16,4.91)$ & 0.948 & $-8.05(-18.68,2.59)$ & 0.051 \\
\hline \multicolumn{7}{|l|}{ Subsample (accelerometer data, $N=1037$ ) } \\
\hline Sedentary time (hours/day) & $-0.11(-2.11,1.90)$ & 0.891 & $1.01(-1.29,3.31)$ & 0.256 & $-3.85(-7.82,0.13)$ & 0.013 \\
\hline Light physical activity (hours/day) & $-0.94(-3.06,1.19)$ & 0.255 & $-1.46(-3.90,0.99)$ & 0.124 & $-0.22(-4.50,4.05)$ & 0.893 \\
\hline Moderate-to-vigorous physical activity (10 min/day) & $0.70(-0.32,1.73)$ & 0.077 & $1.18(0.03,2.33)$ & 0.008 & $-0.23(-2.62,2.17)$ & 0.807 \\
\hline
\end{tabular}

Adjusted for baseline age, sex, socio-economic status, daylight, height and weight z-score, country as a random effect

\section{Effects of adherence to international PA and SB guidelines on $\mathrm{SI}$ percentiles}

At baseline, $17.3 \%$ of the participants with accelerometer data adhered to the PA guideline of at least $1 \mathrm{~h} / \mathrm{d}$ of objectively measured MVPA. When looking at the longitudinal data, only $6.3 \%$ adhered to the guideline at both baseline and two-year follow-up and $4.0 \%$ at both baseline and six-year follow-up. For participants who fulfilled the PA guideline at both time points, there was a higher increase of SI percentiles than for their counterparts with 10.39 units $(p=0.002)$ and 12.68 units $(p=0.050)$ over the two-year and six-year periods, respectively. Meanwhile, $88.8 \%$ of participants adhered to the screen time guideline of watching TV for no more than $14 \mathrm{~h} / \mathrm{w}$ at baseline, $80.6 \%$ adhered to the guidelines at both baseline and twoyear follow-up and $69.6 \%$ at both baseline and six-year follow-up. However, no associations were found between screen time guidelines and SI percentiles.

\section{Discussion}

Our results highlighted the importance of objectively measured MVPA at baseline for the development of a

Table 4 Longitudinal associations between sedentary behaviour, physical activity and bone stiffness index percentiles after 2 years

\begin{tabular}{|c|c|c|c|c|c|c|}
\hline & \multicolumn{2}{|l|}{ Whole group } & \multicolumn{2}{|c|}{ Thin/normal weight group } & \multicolumn{2}{|c|}{ Overweight/obese group } \\
\hline & $\overline{\beta(99 \% \mathrm{Cl})}$ & $p$-value & $\overline{\beta(99 \% \mathrm{Cl})}$ & $\overline{p \text {-value }}$ & $\overline{\beta(99 \% \mathrm{Cl})}$ & $\overline{p \text {-value }}$ \\
\hline \multicolumn{7}{|l|}{ Main sample (self-reported data ${ }_{11} N=1653$ ) } \\
\hline Baseline watching TV/video/DVD (hours/week) & $0.06(-0.33,0.44)$ & 0.712 & $0.28(-0.18,0.74)$ & 0.118 & $-0.63(-1.34,0.07)$ & 0.021 \\
\hline Baseline playing computer/games (hours/week) & $0.02(-0.57,0.60)$ & 0.943 & $-0.12(-0.81,0.57)$ & 0.651 & $0.42(-0.67,1.50)$ & 0.318 \\
\hline Baseline sports clubs (hours/week) & $1.07(-0.23,2.36)$ & 0.034 & $0.96(-0.54,2.46)$ & 0.098 & $1.03(-1.54,3.60)$ & 0.299 \\
\hline Change of watching TV/video/DVD (hours/week) & $0.11(-0.28,0.49)$ & 0.468 & $0.36(-0.10,0.81)$ & 0.042 & $-0.63(-1.35,0.08)$ & 0.022 \\
\hline Change of playing computer/games (hours/week) & $0.09(-0.35,0.54)$ & 0.593 & $0.09(-0.47,0.64)$ & 0.688 & $0.32(-0.41,1.04)$ & 0.258 \\
\hline Change of sports clubs (hours/week) & $1.28(0.30,2.26)$ & 0.001 & $1.29(0.10,2.48)$ & 0.005 & $1.04(-0.63,2.70)$ & 0.110 \\
\hline \multicolumn{7}{|l|}{ Weight bearing sports } \\
\hline $\begin{array}{l}\text { Moderate or high mechanical loads vs. No or low } \\
\text { mechanical loads (reference) }\end{array}$ & $-0.69(-4.99,3.61)$ & 0.678 & $0.32(-4.76,5.41)$ & 0.870 & $-3.13(-11.14,4.89)$ & 0.313 \\
\hline \multicolumn{7}{|l|}{ Subsample (accelerometer data, $N=839$ ) } \\
\hline Baseline sedentary time (hours/day) & $0.60(-1.77,2.96)$ & 0.516 & $0.99(-1.86,3.85)$ & 0.369 & $-0.39(-4.65,3.86)$ & 0.811 \\
\hline Baseline light physical activity (hours/day) & $-0.81(-3.50,1.89)$ & 0.439 & $-1.60(-4.93,1.73)$ & 0.214 & $0.16(-4.46,4.78)$ & 0.927 \\
\hline Baseline moderate-to-vigorous physical activity (10 min/day) & $2.77(1.50,4.05)$ & $<0.001$ & $2.97(1.53,4.42)$ & $<0.001$ & $2.85(-0.12,5.81)$ & 0.013 \\
\hline Change of sedentary time (hours/day) & $-0.85(-2.58,0.88)$ & 0.205 & $-1.19(-3.25,0.87)$ & 0.135 & $0.10(-3.09,3.29)$ & 0.935 \\
\hline Change of light physical activity (hours/day) & $-0.94(-3.03,1.16)$ & 0.249 & $-1.45(-3.99,1.09)$ & 0.140 & $0.42(-3.26,4.10)$ & 0.768 \\
\hline Change of moderate-to-vigorous physical activity (10 min/day) & $1.05(-0.09,2.18)$ & 0.018 & $0.89(-0.39,2.16)$ & 0.073 & $1.76(-0.88,4.40)$ & 0.084 \\
\hline
\end{tabular}

Adjusted for baseline age, sex, socio-economic status, daylight, bone stiffness index percentiles, height and weight z-scores, country as a random effect 
Table 5 Longitudinal associations between sedentary behaviour, physical activity and bone stiffness index percentiles after 6 years

\begin{tabular}{|c|c|c|c|c|c|c|}
\hline & \multicolumn{2}{|l|}{ Whole group } & \multicolumn{2}{|c|}{ Thin/normal weight group } & \multicolumn{2}{|c|}{ Overweight/obese group } \\
\hline & $\beta(99 \% \mathrm{Cl})$ & $p$-value & $\beta(99 \% \mathrm{Cl})$ & $p$-value & $\beta(99 \% \mathrm{Cl})$ & p-value \\
\hline \multicolumn{7}{|l|}{ Main sample (self-reported data, $N=716$ ) } \\
\hline Baseline watching TV/video/DVD (hours/week) & $-0.18(-0.80,0.44)$ & 0.444 & $-0.18(-0.93,0.57)$ & 0.531 & $-0.36(-1.50,0.77)$ & 0.404 \\
\hline Baseline playing computer/games (hours/week) & $0.24(-0.59,1.08)$ & 0.448 & $0.33(-0.73,1.39)$ & 0.420 & $-0.16(-1.51,1.20)$ & 0.766 \\
\hline Baseline sports clubs (hours/week) & $1.79(-0.16,3.74)$ & 0.018 & $1.99(-0.29,4.27)$ & 0.024 & $0.59(-3.20,4.38)$ & 0.686 \\
\hline Change of watching TV/video/DVD (hours/week) & $-0.16(-0.61,0.29)$ & 0.362 & $-0.20(-0.74,0.34)$ & 0.335 & $-0.10(-0.93,0.72)$ & 0.744 \\
\hline Change of playing computer/games (hours/week) & $-0.13(-0.59,0.33)$ & 0.472 & $0.11(-0.45,0.67)$ & 0.617 & $-0.75(-1.58,0.07)$ & 0.019 \\
\hline Change of sports clubs (hours/week) & $1.01(-0.10,2.12)$ & 0.020 & $1.54(0.23,2.85)$ & 0.002 & $-0.46(-2.64,1.73)$ & 0.588 \\
\hline \multicolumn{7}{|l|}{ Weight bearing sports } \\
\hline $\begin{array}{l}\text { Moderate or high mechanical loads vs. No or low } \\
\text { mechanical loads (reference) }\end{array}$ & $4.47(-2.29,11.22)$ & 0.088 & $2.48(-5.39,10.36)$ & 0.416 & $11.26(-1.69,24.22)$ & 0.025 \\
\hline \multicolumn{7}{|l|}{ Subsample (accelerometer data, $N=398$ ) } \\
\hline Baseline sedentary time (hours/day) & $-0.44(-4.00,3.13)$ & 0.751 & $0.08(-4.19,4.35)$ & 0.961 & $1.03(-5.82,7.88)$ & 0.693 \\
\hline Baseline light physical activity (hours/day) & $-2.70(-7.01,1.62)$ & 0.106 & $-1.68(-6.76,3.41)$ & 0.393 & $-5.13(12.46,2.19)$ & 0.068 \\
\hline $\begin{array}{l}\text { Baseline moderate-to-vigorous physical activity } \\
\text { (10 min/day) }\end{array}$ & $3.67(1.55,5.79)$ & $<0.001$ & $3.49(1.03,5.95)$ & $<0.001$ & $4.94(0.92,8.97)$ & 0.002 \\
\hline Change of sedentary time (hours/day) & $-0.18(-2.55,2.18)$ & 0.840 & $0.16(-2.69,3.01)$ & 0.884 & $-1.61(-5.51,2.28)$ & 0.277 \\
\hline Change of light physical activity (hours/day) & $-0.53(-3.78,2.71)$ & 0.670 & $0.11(-3.70,3.92)$ & 0.938 & $-2.18(-7.82,3.45)$ & 0.309 \\
\hline $\begin{array}{l}\text { Change of moderate-to-vigorous physical activity } \\
\text { (10 min/day) }\end{array}$ & $1.53(-0.04,3.10)$ & 0.012 & $1.74(-0.10,3.59)$ & 0.015 & $0.87(-2.09,3.83)$ & 0.441 \\
\hline
\end{tabular}

Adjusted for baseline age, sex, socio-economic status, daylight, bone stiffness index percentiles, height and weight z-scores and puberty at six-year follow-up, country as a random effect

healthy SI over two-year and six-year follow-up. These findings were robust and the effect sizes were consistent across weight statuses. We further demonstrated the benefit of adherence to established PA guidelines on long-term SI gain in children and adolescents, with those participating in objectively measured MVPA for at least $1 \mathrm{~h}$ per day having higher SI increases than their counterparts. These objectively measured results were supported by the comparable, albeit weak positive associations between self-reported time spent at sports clubs and changes in SI percentiles at two-year as well as sixyear follow-ups. Regarding our assumption that being overweight/obese may be an important confounder, we observed controversial associations of screen-based SB with SI in the different weight strata. In general, the inversely cross-sectional associations between watching $\mathrm{TV}$ and SI were more pronounced in thin/normal weight children and adolescents than in overweight/obese ones. Nonetheless, in the longitudinal data, durations of specific screen-based SB were observed to be negatively associated with SI changes only in overweight/obese participants at the two-year and six-year follow-ups.

Even though the beneficial osteogenic effect of PA on bone mass accrual has already been well described in previous observational studies, most of the existing evidence so far mainly focused on BMC and/or BMD [51, 52]. Only a few cross-sectional studies have examined the associations between PA and QUS bone parameters. For example, Robinson et al. [53] demonstrated that time spent on moderate-to-high impact activities positively related to calcaneus SI in adolescent girls. Zulfarina et al. [54] reported that PA level, in terms of metabolic equivalent-minutes per week, was positively associated with three QUS parameters (i.e. BUA, SOS and SI) in adolescents. However, the PA levels in previous studies were mainly measured using different selfreported questionnaires, rendering it difficult to compare the results. Moreover, little is known about the optimal dose and intensity of PA and their sustainable effects on bone strength during growth. In our previous casecontrol study that was embedded in the IDEFICS study, we found that $30 \mathrm{~min}$ of objectively measured MVPA per day was not sufficient for an optimal SI [55]. A longitudinal study suggested that children in the upper quartile of objectively measured MVPA (approximated $1 \mathrm{~h} / \mathrm{d}$ ) had about 4 to $13 \%$ greater HR-pQCT-measured bone parameters at distal tibia compared to their peers in the lowest quartile (approximated $0.5 \mathrm{~h} / \mathrm{d}$ ) [56]. Our findings not only demonstrate that objectively measured MVPA rather than LPA using accelerometers is an important predictor of bone strength across weight strata, but also support the current opinion that adherence to the WHO recommendations for MVPA has a positive impact on bone strength. A recent systematic review 
suggested that more than $80 \%$ of adolescents had insufficient physical activity globally illustrating the urgent need for further effective policies and intervention strategies in order to obtain optimal bone strength in children and adolescents [57].

Regarding self-reported PA at baseline, less than half of the parents reported that their children participated in a sports club. The average time spent at sports clubs was given as approximately $1 \mathrm{~h}$ per week. This indeed depicts reality, as children of that age do not commonly take part in sports club activities. Our finding of change in time spent at sports clubs rather than baseline time being more strongly related to change in SI was hence to be expected, as the participants were then 2 and 6 years older at the respective follow-ups. While no association between self-reported WBE and SI was observed in our study, the osteogenic effect of WBE has been reported in a review regarding school-based intervention programs, however, focusing mainly on jumping exercises [58]. Bone strength is thought to be less sensitive to light and moderate WBE among growing individuals [59]. As we did not have data describing the intensity of WBE in our study this may possibly explain why our results were non-significant. Further, as the self-reported sports club activities did not include WBE during leisure time, the effect of WBE on SI may have been underestimated.

Notably, the cross-sectional associations we observed did not match the longitudinal associations across weight strata, especially in self-reported screen-based SB. A possible explanation could be that the deleterious effects of watching TV on SI were covered by the stimulating effect of the mechanical loading exerted by weight status, which increased along with more screen time [60]. However, after relatively long-term exposure to screen-based SB, less SI gain still could occur in overweight/obese children. Potential direct and indirect mechanisms of weight status may influence the longitudinal relationship between screen-based SB and SI development. On the one hand, being sedentary may disrupt the bone formation-resorption balance due to lack of mechanical loading [61]. This detrimental effect may be stronger in overweight/obese individuals since they lose more mechanical loading exerted by body weight. On the other hand, overweight/obese children are also likely to spend more time watching TV or playing computer/ games [62], eventually resulting in reduced SI. A similar observation was made in our data. Moreover, detrimental effects of screen-based SB appeared to be patternspecific over time, with the durations of watching TV and playing computer/games observed to be inversely associated with SI gain at two-year and six-year followup, respectively. Although watching TV, the predominantly measured screen-based SB, represents the largest amount of screen-based SB for most children, recent studies suggest that computer use has increased dramatically over the years and has even replaced time spent watching TV, especially in adolescents [63, 64]. Marco et al. [17] also reported that non-study internet use rather than watching TV was negatively associated with whole body BMC in male adolescents. Our results are in line with this behavioural transition from childhood to adolescence and demonstrate that playing computer/ games might present a higher risk factor for bone strength than watching TV as children get older.

The relationship between objectively measured SB and bone health during growth is still inconclusive. Results of a recent systematic review indicated the presence of a minor association between total SB and bone outcomes of the lower extremities in youth [65]. In the British Columbia Healthy Bones Study III cohort (HBSII), no associations were found between screen-based $\mathrm{SB}$, total SB and bone architecture and strength in 9- to 20-yearold subjects at baseline [16], but total duration of objectively measured SB was found to be a negatively independent predictor in longitudinal analyses based on four annual follow-ups [56]. In contrast, we did not observe any longitudinal relationships between total duration of objectively measured SB and SI, except for a small cross-sectional inverse association in overweight/ obese children. In our previous IDEFICS study with a larger cross-sectional sample, we found that total duration of objectively measured SB was negatively associated with SI in preschool and school children [26]. As only a fraction of the subgroup of participants in the present study who had accelerometer data could be linked in longitudinal data, we believe that the associations we detected did not reach the significance threshold due to the small sample size. Nevertheless, from our investigation, it still can be concluded that SB operationalised as screen time might be a valuable predictor of bone strength. However, the optimal dose of SB as well as of specific screen-based SB on bone strength needs to be further investigated in longitudinal studies and interventions.

Our study has several strengths and limitations. To our best knowledge, this is the first longitudinal study to present the associations between SB, PA and QUS parameters using repeatedly measured data among European children and adolescents. Moreover, in addition to self-reported questionnaires, we investigated SB and PA with objective measurements in a relatively large subsample and thereby acquired more precise information regarding the intensity and quantification of activity levels. Additionally, we were able to identify differential associations of SI across weight strata, which helped provide better insight into the role of weight status in these associations. While we were able to collect some 
objective data, the fact that the data was only available for a subsample whose size diminished considerably at each follow-up is a limitation that most likely led to the lack of statistical power, and our results may not be generalised for the whole population. However, as the subsample's baseline mean of SI percentiles $(41.19 \pm 26.58)$ and rate of overweight/obesity (19.7\%) were comparable to that of the main sample $(43.44 \pm 27.47$ and $19.0 \%$, respectively), we believe that this reduces the potential for bias. Second, the imprecision of the self-reported WBE (only based on sports club activities) may have led to underestimations regarding cross-sectional and longitudinal effects of WBE on SI. Third, the comparability of ActiTrainer with other Actigraph accelerometers in previous validation studies is still inconclusive. However, we additionally included a confounder to account for different measures induced by the use of GT1M and ActiTrainer, which did not change our final results. Therefore, we are convinced that our data collection by different types of accelerometers provides comparable PA values. Finally, we did not include nutritional variables such as calcium intake. Instead, we considered weekly frequency of milk and dairy products consumption from a food frequency questionnaire as a proxy. Since we did not observe an influential effect on our final results, we did not consider this variable further to avoid having to exclude more participants, which would have reduced the sample size considerably.

\section{Conclusions}

In summary, our results demonstrated that objectively measured MVPA is an important predictor of bone strength across weight strata. Meeting the MVPA recommendation of $1 \mathrm{~h}$ per day maintained the beneficial effect on bone strength during the six-year observational period. On the other hand, the increasing durations of screen-based SB might be risk factors for SI development, especially in overweight/obese participants. Finally, bone health improving interventions should promote high intensive exercises and also focus on the reduction of screen-based SB, particularly when targeting overweight/ obese individuals.

\section{Abbreviations \\ PBM: Peak bone mass; PA: Physical activity; SB: Sedentary behaviour; WBE: Weight-bearing exercises; WHO: World Health Organization; LPA: Light physical activity; MVPA: Moderate-to-vigorous physical activity; QUS: Quantitative ultrasound; SI: Stiffness index; BUA: Broadband ultrasound attenuation; SOS: Speed of sound; BMC: Bone mineral content; BMD: Bone mineral density; DXA: Dual energy X-ray absorptiometry; BMI: Body mass index; IOTF: International Obesity Task Force; CPM: Counts per minute; SES: Socio-economic status; ISCED: International Standard Classification of Education; SD: Standard deviation; Cl: Confidence interval}

\section{Acknowledgements}

We gratefully acknowledge all the children and their parents for participating in the IDEFICS/I. Family studies. We appreciate the support from schools, communities and the entire IDEFICS/ I. Family study teams. We appreciate the kindly help in English language editing from our colleagues Dr. Florence Samkange-Zeeb and Heike Gerds. L.C. personally wishes to thank the China Scholarship Council (CSC) for the financial support for her PhD study.

\section{Authors' contributions}

L.C., W.A. and A.H. conceptualised and designed the study. L.C., H.P. and A.H. analysed and interpreted the data. W.A., F.L., T.V., C.C., D.M., G.E., N.M., L.M., A.P. and Y.P. contributed to coordination and data collection. L.C. was a major contributor in writing the manuscript. All authors read and improved the manuscript and approved the final manuscript.

\section{Funding}

This study as part of the IDEFICS and I. Family studies was financially supported by the European Commission within the Sixth RTD Framework Programme Contract No.016181 (FOOD) and the Seventh RTD Framework Programme Contract No.266044, respectively. The funding body was not involved in the study design, data collection, analysis and interpretation as well as manuscript writing.

\section{Availability of data and materials}

The datasets generated and analysed during the current study are not publicly available because this study is based on highly sensitive data collected in young children. But interested researchers can contact the IDEFICS and I. Family consortia (http://www.ideficsstudy.eu/ldefics/ and http://www.ifamilystudy.eu/) to discuss possibilities for data access.

\section{Ethics approval and consent to participate}

All parents and their children above 12 years old signed informed consent, while younger children gave oral consent prior to the examinations in addition to the signed parental consent. Ethical approval was obtained from the ethics committees for all participating centres in each country: Ethics Committee, University Hospital, Gent, Belgium; Cyprus National Bioethics Committee, Nicosia, Cyprus; Tallinn Medical Research Ethics Committee, Tallinn, Estonia; Ethics Committee of the University of Bremen, Bremen, Germany; Egészségügyi Tudományos Tanács, Pécs, Hungary; Azienda Sanitaria Locale Avellino Comitato Etico, Avellino, Italy; Regionala Etikprövningsnämnden i Göteborg, Gothenburg, Sweden; Comité Ético de Investigación Clínica de Aragón, Zaragoza, Spain.

Consent for publication

Not applicable.

\section{Competing interests}

The authors declare that they have no competing interests.

\section{Author details}

${ }^{1}$ Leibniz Institute for Prevention Research and Epidemiology - BIPS, Achterstraße 30, 28359 Bremen, Germany. ${ }^{2}$ Faculty of Mathematics and Computer Science, University of Bremen, Bremen, Germany. ${ }^{3}$ Institute of Food Sciences, National Research Council, Avellino, Italy. ${ }^{4}$ Department of Chronic Diseases, National Institute for Health Development, Tallinn, Estonia. ${ }^{5}$ Research and Education Institute of Child Health, Strovolos, Cyprus.

${ }^{6}$ Department of Pediatrics, Medical School, University of Pécs, Pécs, Hungary. ${ }^{7}$ Department of Public Health, School of Health Sciences, University of Skövde, Skövde, Sweden. ${ }^{8}$ Department of Public Health, Faculty of Medicine and Health Sciences, Ghent University, 9000 Ghent, Belgium. ${ }^{9}$ GENUD (Growth, Exercise, Nutrition and Development) Research Group, Instituto Agroalimentario de Aragón (IA2), Instituto de Investigación Sanitaria Aragón (IIS Aragón), Centro de Investigación Biomédica en Red Fisiopatología de la Obesidad y Nutrición (CIBERObn), University of Zaragoza, 50009 Zaragoza, Spain. ${ }^{10}$ Centre for Exercise, Nutrition \& Health Sciences, University of Bristol, Bristol, UK. ${ }^{11}$ Collaborating Centre of Sports Medicine, University of Brighton, Brighton, UK.

Received: 23 July 2019 Accepted: 13 April 2020

Published online: 28 April 2020

\section{References}

1. Bachrach LK. Acquisition of optimal bone mass in childhood and adolescence. Trends Endocrinol Metab. 2001;12(1):22-8. 
2. Nguyen TV, Maynard LM, Towne B, Roche AF, Wisemandle W, Li J, et al. Sex differences in bone mass acquisition during growth: the Fels longitudinal study. J Clin Densitom. 2001;4(2):147-57.

3. Mitchell JA, Cousminer DL, Zemel BS, Grant SF, Chesi A. Genetics of pediatric bone strength. Bonekey Rep. 2016;5:823.

4. Gordon CM, Zemel BS, Wren TA, Leonard MB, Bachrach LK, Rauch F, et al. The determinants of peak bone mass. J Pediatr. 2017;180:261-9.

5. Julian-Almarcegui C, Gomez-Cabello A, Huybrechts I, Gonzalez-Aguero A, Kaufman JM, Casajus JA, et al. Combined effects of interaction between physical activity and nutrition on bone health in children and adolescents: a systematic review. Nutr Rev. 2015;73(3):127-39.

6. Seeman E. Reduced bone formation and increased bone resorption: rational targets for the treatment of osteoporosis. Osteoporos Int. 2003;14(Suppl 3): S2-8.

7. Behringer M, Gruetzner S, McCourt M, Mester J. Effects of weight-bearing activities on bone mineral content and density in children and adolescents: a meta-analysis. J Bone Miner Res. 2014;29(2):467-78.

8. Tan VP, Macdonald HM, Kim S, Nettlefold L, Gabel L, Ashe MC, et al. Influence of physical activity on bone strength in children and adolescents: a systematic review and narrative synthesis. J Bone Miner Res. 2014;29(10): 2161-81.

9. Konstabel K, Veidebaum T, Verbestel V, Moreno LA, Bammann K, Tornaritis $M$, et al. Objectively measured physical activity in European children: the IDEFICS study. Int J Obes. 2014;38(Suppl 2):S135-43.

10. Marques A, Gaspar de Matos M. Adolescents' physical activity trends over the years: a three-cohort study based on the health behaviour in schoolaged children (HBSC) Portuguese survey. BMJ Open. 2014;4(9):e006012.

11. Sigmund E, Sigmundova D, Badura P, Kalman M, Hamrik Z, Pavelka J. Temporal trends in overweight and obesity, physical activity and screen time among Czech adolescents from 2002 to 2014: a National Health Behaviour in school-aged children study. Int J Environ Res Public Health. 2015:12(9):11848-68

12. Carson V, Hunter S, Kuzik N, Gray CE, Poitras VJ, Chaput JP, et al. Systematic review of sedentary behaviour and health indicators in school-aged children and youth: an update. Appl Physiol Nutr Metab. 2016;41(6 Suppl 3): S240-65.

13. Verloigne M, Van Lippevelde W, Maes L, Yildirim M, Chinapaw M, Manios Y, et al. Self-reported TV and computer time do not represent accelerometerderived total sedentary time in 10 to 12-year-olds. Eur J Pub Health. 2013; 23(1):30-2.

14. Brug J, van Stralen MM, Te Velde SJ, Chinapaw MJ, De Bourdeaudhuij I, Lien $\mathrm{N}$, et al. Differences in weight status and energy-balance related behaviors among schoolchildren across Europe: the ENERGY-project. PLoS One. 2012:7(4):e34742.

15. Sioen I, Michels N, Polfliet C, De Smet S, D'Haese S, Roggen I, et al. The influence of dairy consumption, sedentary behaviour and physical activity on bone mass in Flemish children: a cross-sectional study. BMC Public Health. 2015;15:717.

16. Gabel L, McKay HA, Nettlefold L, Race D, Macdonald HM. Bone architecture and strength in the growing skeleton: the role of sedentary time. Med Sci Sports Exerc. 2015;47(2):363-72.

17. Gracia-Marco L, Rey-Lopez JP, Santaliestra-Pasias AM, Jimenez-Pavon D, Diaz $L E$, Moreno LA, et al. Sedentary behaviours and its association with bone mass in adolescents: the HELENA cross-sectional study. BMC Public Health. 2012;12:971.

18. McVeigh JA, Zhu K, Mountain J, Pennell CE, Lye SJ, Walsh JP, et al. Longitudinal trajectories of television watching across childhood and adolescence predict bone mass at age 20 years in the Raine study. J Bone Miner Res. 2016;31(11):2032-40.

19. Winther A, Ahmed LA, Furberg AS, Grimnes G, Jorde R, Nilsen OA, et al. Leisure time computer use and adolescent bone health--findings from the Tromso study, fit futures: a cross-sectional study. BMJ Open. 2015;5(6): e006665.

20. NCD Risk Factor Collaboration. Worldwide trends in body-mass index underweight, overweight, and obesity from 1975 to 2016: a pooled analysis of 2416 population-based measurement studies in 128.9 million children, adolescents, and adults. Lancet. 2017;390(10113):2627-42.

21. van Leeuwen J, Koes BW, Paulis WD, van Middelkoop M. Differences in bone mineral density between normal-weight children and children with overweight and obesity: a systematic review and meta-analysis. Obes Rev. 2017:18(5):526-46
22. Wetzsteon RJ, Petit MA, Macdonald HM, Hughes JM, Beck TJ, McKay HA. Bone structure and volumetric BMD in overweight children: a longitudinal study. J Bone Miner Res. 2008;23(12):1946-53.

23. Tanaka C, Janssen X, Pearce M, Parkinson K, Basterfield L, Adamson A, et al. Bidirectional associations between adiposity, sedentary behavior, and physical activity: a longitudinal study in children. J Phys Act Health. 2018:7:1-9.

24. Ahrens W, Bammann K, Siani A, Buchecker K, De Henauw S, lacoviello L, et al. The IDEFICS cohort: design, characteristics and participation in the baseline survey. Int J Obes. 2011;35(Suppl 1):S3-15.

25. Ahrens W, Siani A, Adan R, De Henauw S, Eiben G, Gwozdz W, et al. Cohort Profile: The transition from childhood to adolescence in European childrenhow I. Family extends the IDEFICS cohort. Int J Epidemiol. 2017;46(5):1394-5j.

26. Herrmann D, Buck C, Sioen I, Kouride Y, Marild S, Molnar D, et al. Impact of physical activity, sedentary behaviour and muscle strength on bone stiffness in 2-10-year-old children-cross-sectional results from the IDEFICS study. Int J Behav Nutr Phys Act. 2015;12:112.

27. Baroncelli Gl. Quantitative ultrasound methods to assess bone mineral status in children: technical characteristics, performance, and clinical application. Pediatr Res. 2008;63(3):220-8.

28. Alwis G, Rosengren B, Nilsson JA, Stenevi-Lundgren S, Sundberg M, Sernbo I, et al. Normative calcaneal quantitative ultrasound data as an estimation of skeletal development in Swedish children and adolescents. Calcif Tissue Int. 2010;87(6):493-506.

29. Torres-Costoso A, Vlachopoulos D, Ubago-Guisado E, Ferri-Morales A, Cavero-Redondo I, Martinez-Vizcaino V, et al. Agreement between dualenergy $\mathrm{X}$-ray absorptiometry and quantitative ultrasound to evaluate bone health in adolescents: the PRO-BONE study. Pediatr Exerc Sci. 2018;30(4): 466-73.

30. Trimpou P, Bosaeus I, Bengtsson BA, Landin-Wilhelmsen K. High correlation between quantitative ultrasound and DXA during 7 years of follow-up. Eur J Radiol. 2010;73(2):360-4.

31. Sioen I, Mouratidou T, Herrmann D, De Henauw S, Kaufman JM, Molnar D, et al. Relationship between markers of body fat and calcaneal bone stiffness differs between preschool and primary school children: results from the IDEFICS baseline survey. Calcif Tissue Int. 2012;91 (4):276-85.

32. Herrmann D, Intemann T, Lauria F, Marild S, Molnar D, Moreno LA, et al. Reference values of bone stiffness index and C-terminal telopeptide in healthy European children. Int J Obes. 2014;38(Suppl 2):S76-85.

33. Stomfai S, Ahrens W, Bammann K, Kovacs E, Marild S, Michels N, et al. Intraand inter-observer reliability in anthropometric measurements in children. Int J Obes. 2011;35(Suppl 1):S45-51.

34. Cole TJ, Freeman JV, Preece MA. British 1990 growth reference centiles for weight, height, body mass index and head circumference fitted by maximum penalized likelihood. Stat Med. 1998;17(4):407-29.

35. Cole TJ, Lobstein T. Extended international (IOTF) body mass index cut-offs for thinness, overweight and obesity. Pediatr Obes. 2012;7(4):284-94.

36. Tremblay MS, Carson V, Chaput JP, Connor Gorber S, Dinh T, Duggan M, et al. Canadian 24-hour movement guidelines for children and youth: an integration of physical activity, sedentary behaviour, and sleep. Appl Physiol Nutr Metab. 2016;41(6 Suppl 3):S311-27.

37. American Academy of Pediatrics. Committee on Public E. American Academy of Pediatrics: children, adolescents, and television. Pediatrics. 2001; 107(2):423-6.

38. Lee KY, Macfarlane DJ, Cerin E. Comparison of three models of actigraph accelerometers during free living and controlled laboratory conditions. Eur J Sport Sci. 2013;13(3):332-9.

39. Santos-Lozano A, Santin-Medeiros F, Cristi-Montero C, Jaen-Jimenez R, Casajus JA, Garatachea N. GT1M, GT3X and ActiTrainer counts comparison during standardized activities in young, adults and older adults. Nutr Hosp. 2016;33(3):280.

40. Sasaki JE, John D, Freedson PS. Validation and comparison of ActiGraph activity monitors. J Sci Med Sport. 2011;14(5):411-6.

41. Robusto KM, Trost SG. Comparison of three generations of ActiGraph activity monitors in children and adolescents. J Sports Sci. 2012;30(13):1429-35.

42. Trost SG, Loprinzi PD, Moore R, Pfeiffer KA. Comparison of accelerometer cut points for predicting activity intensity in youth. Med Sci Sports Exerc. 2011:43(7):1360-8.

43. Buck C, Eiben G, Lauria F, Konstabel K, Page A, Ahrens W, et al. Urban Moveability and physical activity in children: longitudinal results from the IDEFICS and I. Family cohort. Int J Behav Nutr Phys Act. 2019; 16(1):128 
44. Harvey NC, Cole ZA, Crozier SR, Kim M, Ntani G, Goodfellow L, et al. Physical activity, calcium intake and childhood bone mineral: a population-based cross-sectional study. Osteoporos Int. 2012;23(1):121-30.

45. WHO. Global recommendations on physical activity for health. 2010.

46. UNESCO. International Standard Classification of Education 2010. http:// www.uis.unesco.org/Education/Pages/international-standardclassification-ofeducation.aspx.

47. Harries M, Hawkins S, Hacking J, Hughes I. Changes in the male voice at puberty: vocal fold length and its relationship to the fundamental frequency of the voice. J Laryngol Otol. 1998;112(5):451-4.

48. Harries ML, Walker JM, Williams DM, Hawkins S, Hughes IA. Changes in the male voice at puberty. Arch Dis Child. 1997;77(5):445-7.

49. Lawn RB, Lawlor DA, Fraser A. Associations between maternal Prepregnancy body mass index and gestational weight gain and Daughter's age at menarche: the Avon longitudinal study of parents and children. Am 」 Epidemiol. 2018;187(4):677-86.

50. Moon RJ, Davies JH, Cooper C, Harvey NC. Vitamin D, and maternal and child health. Calcif Tissue Int. 2020;106(1):30-46.

51. Heidemann M, Molgaard C, Husby S, Schou AJ, Klakk H, Moller NC, et al. The intensity of physical activity influences bone mineral accrual in childhood: the childhood health, activity and motor performance school (the CHAMPS) study, Denmark. BMC Pediatr. 2013;13:32.

52. Janz KF, Gilmore JM, Levy SM, Letuchy EM, Burns TL, Beck TJ. Physical activity and femoral neck bone strength during childhood: the lowa bone development study. Bone. 2007;41(2):216-22.

53. Robinson ML, Winters-Stone K, Gabel K, Dolny D. Modifiable lifestyle factors affecting bone health using calcaneus quantitative ultrasound in adolescent girls. Osteoporos Int. 2007;18(8):1101-7.

54. Zulfarina MS, Sharif R, Syarifah-Noratiqah SB, Sharkawi AM, Aqilah-Sm ZS Mokhtar SA, et al. Modifiable factors associated with bone health in Malaysian adolescents utilising calcaneus quantitative ultrasound. PLoS One. 2018;13(8):e0202321.

55. Herrmann D, Pohlabeln H, Gianfagna F, Konstabel K, Lissner L, Marild S, et al. Association between bone stiffness and nutritional biomarkers combined with weight-bearing exercise, physical activity, and sedentary time in preadolescent children. A case-control study. Bone. 2015;78:142-9.

56. Gabel L, Macdonald HM, Nettlefold L, McKay HA. Physical activity, sedentary time, and bone strength from childhood to early adulthood: a mixed longitudinal HR-pQCT study. J Bone Miner Res. 2017;32(7):1525-36.

57. Guthold R, Stevens GA, Riley LM, Bull FC. Global trends in insufficient physical activity among adolescents: a pooled analysis of 298 populationbased surveys with 1.6 million participants. Lancet Child Adolesc Health. 2020;4(1):23-35.

58. Nguyen $\mathrm{VH}$. School-based exercise interventions effectively increase bone mineralization in children and adolescents. Osteoporos Sarcopenia. 2018; 4(2):39-46.

59. Sayers A, Mattocks C, Deere K, Ness A, Riddoch C, Tobias JH. Habitual levels of vigorous, but not moderate or light, physical activity is positively related to cortical bone mass in adolescents. J Clin Endocrinol Metab. 2011;96(5):E793-802.

60. Keane E, Li X, Harrington JM, Fitzgerald AP, Perry IJ, Kearney PM. Physical activity, sedentary behavior and the risk of overweight and obesity in school-aged children. Pediatr Exerc Sci. 2017;29(3):408-18.

61. Tremblay MS, Colley RC, Saunders TJ, Healy GN, Owen N. Physiological and health implications of a sedentary lifestyle. Appl Physiol Nutr Metab. 2010; 35(6):725-40.

62. Stierlin AS, De Lepeleere S, Cardon G, Dargent-Molina P, Hoffmann B, Murphy MH, et al. A systematic review of determinants of sedentary behaviour in youth: a DEDIPAC-study. Int J Behav Nutr Phys Act. 2015;12:133.

63. Huhman M, Lowry R, Lee SM, Fulton JE, Carlson SA, Patnode CD. Physical activity and screen time: trends in U.S. children aged 9-13 years, 2002-2006. J Phys Act Health. 2012;9(4):508-15.

64. Sigmundova D, Sigmund E, Bucksch J, Badura P, Kalman M, Hamrik Z. Trends in screen time Behaviours in Czech schoolchildren between 2002 and 2014: HBSC study. Cent Eur J Public Health. 2017;25(Suppl 1):S15-20

65. Koedijk JB, van Rijswijk J, Oranje WA, van den Bergh JP, Bours SP, Savelberg $\mathrm{HH}$, et al. Sedentary behaviour and bone health in children, adolescents and young adults: a systematic review. Osteoporos Int. 2017;28(9):2507-19.

\section{Publisher's Note}

Springer Nature remains neutral with regard to jurisdictional claims in published maps and institutional affiliations.

\section{Ready to submit your research? Choose BMC and benefit from}

- fast, convenient online submission

- thorough peer review by experienced researchers in your field

- rapid publication on acceptance

- support for research data, including large and complex data types

- gold Open Access which fosters wider collaboration and increased citations

- maximum visibility for your research: over $100 \mathrm{M}$ website views per year

At BMC, research is always in progress.

Learn more biomedcentral.com/submissions 